\title{
Orientation of molecules with a small dipole moment using cyclic wave packets
}

\author{
Juan Ortigoso and Julio Santos \\ Instituto de Estructura de la Materia, CSIC, Serrano 121, 28006 Madrid, Spain \\ (Received 15 April 2005; published 4 November 2005)
}

\begin{abstract}
A robust and versatile method is demonstrated to maintain, for an arbitrary time, quasiconstant and strong orientation for molecules with minute dipole moments. The procedure is based on submitting molecules in cyclic wave packets to periodic trains of nonresonant pulses in an electrostatic field. We show that approximate cyclic states can be created by suddenly switching off a long laser pulse. A control scheme to remove the static field without spoiling orientation is suggested, which would permit large field-free orientation between pulses.

DOI: 10.1103/PhysRevA.72.053401

PACS number(s): 32.80.Lg, 33.15.Kr, 33.80.-b
\end{abstract}

\section{INTRODUCTION}

The creation of durable molecular orientation and/or alignment is becoming a very active topic of molecular physics [1]. Polar molecules can interact with a static electric field giving rise to pendular states, which execute a small amplitude libration about the field direction [2]. Linear or diatomic molecules traveling in a molecular beam evolve adiabatically while entering into the field, due to the absence of sharp avoided crossings between Stark energy levels. Therefore molecules are described, inside the field, by a single pendular state. For large values of the dimensionless ratio $\omega_{S} \equiv \mu E_{S} / B$ ( $\mu$ is the permanent dipole moment, $E_{S}$ the electric field, and $B$ the rotational constant) the lowest pendular states are strongly oriented. Thus, lasting and strong orientation can be easily achieved. For the more complex case of asymmetric-top molecules nonadiabatic evolution, due to the edge effects of the static field, is possible and a complex dynamical behavior occurs [3].

Friedrich and Herschbach [4] realized that molecular orientation is enhanced when a congruent combination of a static field and an intense nonresonant laser pulse is employed. The interaction of molecular polarizability with the nonresonant laser creates a double well potential where aligned tunneling doublets exist. An additional electrostatic field can mix these doublets giving rise to a pseudo-firstorder Stark effect that creates strongly oriented states [4]. Successful experimental demonstrations of this method have been published [5].

Other techniques that can convert alignment into orientation have been proposed. They share the requisite of breaking the parity of the wave function by mixing even and odd rotational levels. For example, two overlapping lasers can orient molecules by adiabatic passage along dressed states [6].

Similarly to the alignment dynamics of a molecule interacting with a nonresonant pulse [7] different regimes, from fully adiabatic to nonadiabatic, are possible for the orientation dynamics when a static field is added [8]. In the adiabatic case, the molecule is oriented during the pulse. After the pulse dies it is described by a single Stark eigenstate, which, for small $\omega_{S}$, is only weakly oriented. In the nonadiabatic case post-pulse recurrences in the orientation occur since the molecule ends up in a linear combination of several Stark states. Thus, in general, large orientation cannot be maintained with this method. Nonetheless, the use of nanosecond pulses offers a route to conserve strong orientation, inside a electrostatic field, for a time that may suffice for many applications [4].

Other strategies have been developed to orient molecules under field-free conditions. They are especially important, since numerous experiments require field-free transient orientation. These methods involve different theoretical and technological approaches such as two-color phase-locked laser excitation [9], half-cycle pulses [10,11], sudden and asymmetric laser pulses (containing a given frequency and its second harmonic) [12], hybrid pulses formed by a halfcycle pulse and a nonresonant laser [13], etc. Another interesting method takes advantage of the rovibrational dynamics upon laser excitation to an excited electronic state, to create a situation that simulates the interaction of a molecule with a pulse train in a static field [14]. Also, the use of optimized trains of nonresonant pulses can enhance field-free alignment [15].

Here, we present a strategy to maintain, for molecules with a tiny dipole moment, and during an arbitrary time, nearly perfect molecular orientation $(\langle\cos \theta\rangle \geqslant 0.9$, where $\theta$ is the angle between the molecular axis and the field direction). The scheme is based on the idea that special properties of rotational wave packets, corresponding to eigenstates of a Floquet-type operator (cyclic states), are conserved when they are submitted to a periodic sequence of properly timed nonresonant laser pulses [16].

The paper is organized as follows. In Sec. II we describe briefly the basic characteristics of cyclic states in the context of the $\left(t, t^{\prime}\right)$ method [17], and show that, for a molecule in a static field combined with a train of laser pulses, some of them are highly oriented. Conditions under which conservation of large orientation is obtained are discussed. A crucial step of our scheme is the preparation of the initial state. We show, in Sec. III, that rotational wave packets sufficiently similar to exact cyclic states can be easily created by a slight modification of the switched-wave-packet method $[18,19]$. Thus, our method is robust since orientation is conserved for these approximate cyclic states. In Sec. IV we discuss a control scheme for conserving large orientation upon removal of the electrostatic field. A new consequence of this procedure is the possibility of obtaining periodically, in a controlled way, effective field-free orientation for molecules with a very 
small dipole moment. Finally we summarize our main conclusions in Sec. V.

\section{ORIENTED CYCLIC STATES FOR A PULSE TRAIN COMBINED WITH A STATIC FIELD}

In the $\left(t, t^{\prime}\right)$ formalism [17] the time variable in the Hamiltonian is considered as an extra coordinate, $t^{\prime}$. Solutions to the time-dependent Schrödinger equation can be formally written as:

$$
\psi(t)=\left.\exp \left[(-i / \hbar) S\left(t^{\prime}\right)\left(t-t_{0}\right)\right] \psi\left(t^{\prime}, t_{0}\right)\right|_{t^{\prime}=t},
$$

where the Schrödinger operator $S\left(t^{\prime}\right)=-i \hbar \partial / \partial t^{\prime}+H\left(t^{\prime}\right)$ is time independent. Thus, the Schrödinger equation can be solved, in this formalism, by using methods developed for time-independent problems.

The operator $S\left(t^{\prime}\right)$, in dimensionless form (which suggests to use $\hbar / B$ as a reduced unit of time and $B / \hbar$ as a reduced unit of frequency), for a linear molecule in the presence of a static field and a linearly-polarized nonresonant laser pulse with their field directions parallel to each other, is

$$
S\left(t^{\prime}\right)=-i \frac{\hbar}{B} \frac{\partial}{\partial t^{\prime}}+\mathbf{J}^{2}-\omega_{S} \cos \theta-\left(\Delta \omega \cos ^{2} \theta+\omega_{\perp}\right) g\left(t^{\prime}\right),
$$

where $\mathbf{J}$ is the angular momentum operator, $\Delta \omega=\omega_{\|}-\omega_{\perp}$, and $\omega_{\|, \perp}=\alpha_{\|, \perp} E_{L}^{2} /(4 B)$. The constants $\alpha_{\|}$, and $\alpha_{\perp}$ are the parallel and perpendicular components of molecular polarizability, and $E_{L}$ the strength of the laser field. The pulse shape is taken to be a Gaussian centered at $t^{\prime}=0, g\left(t^{\prime}\right)$ $=\exp \left(-t^{\prime 2} / \sigma^{2}\right)$. Eigenstates of $S\left(t^{\prime}\right)$ can be expanded as $\left|\lambda\left(t^{\prime}\right)\right\rangle=\sum_{J, n} c_{J n}|J\rangle|n\rangle$, where $|J\rangle$ are rotational eigenstates, and $|n\rangle=\exp \left(2 \pi i n t^{\prime} / T\right) / \sqrt{T}$ Fourier basis functions for the time coordinate. The period, $T$, represents physically the delay between pulses in a periodic pulse train [16] and can be freely chosen. In the case of a molecule interacting with a single pulse, this treatment implies to consider that the pulse is repeated periodically with period $T$, which is fully justified for high-frequency lasers [17].

Eigenstates of $S\left(t^{\prime}\right)$ are cyclic [20]. This means that if the wave function at $t_{0}=-T / 2$ is chosen as the projection onto the spatial (rotational) Hilbert state of just one eigenstate, $\quad \psi\left(t_{0}\right)=\left.\lambda\left(t^{\prime}\right)\right|_{t^{\prime}=t_{0}}=\Sigma_{J} c_{J}^{\prime}\left(t_{0}\right)|J\rangle$, where $c_{J}^{\prime}\left(t_{0}\right)=\Sigma_{n} c_{J n}$ $\times \exp (-i n \pi) / \sqrt{T}$, its evolution at the end of pulse number $l$ of a periodic pulse train is given simply by $\exp \left(-i \epsilon_{\lambda} l T B / \hbar\right) \psi\left(t_{0}\right)$, where $\epsilon_{\lambda}$ is the eigenvalue corresponding to $|\lambda\rangle$. Thus, the initial state is recovered, at the end of each pulse, except for a global phase factor that does not affect the orientation.

One of us recently showed that some cyclic states for a molecule interacting with a periodic train of nonresonant pulses remain well aligned [16]. If close lying pairs of aligned cyclic states with different parity exist, the addition of a weak static field could transform alignment into orientation. The closer the aligned cyclic states the weaker the static field needed to obtain oriented cyclic states. In order to maximize the dipole coupling between a pair of quasidegen-

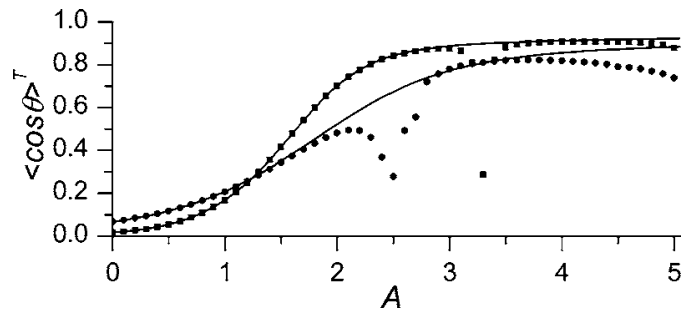

FIG. 1. The average orientation over one period as a function of pulse area. Dots correspond to cyclic states of Eq. (2) (squares are for $\sigma=0.01, T=0.1, \omega_{S}=0.05$, and circles for $\sigma=0.02, T=0.2, \omega_{S}$ $=0.2$ ). The continuous lines correspond to pendular states of Eq. (4).

erate aligned cyclic states they should be ideally composed of rotational eigenstates with low $J$. Thus, further dephasing is also minimized [21]. This mechanism, for cyclic states, is similar to the one described in [4] for instantaneous eigenstates of a molecule in a long nonresonant pulse.

The dependence of orientation for cyclic states as a function of the Hamiltonian parameters can be understood by using a time-independent perturbative expansion of $S\left(t^{\prime}\right)$ [22]:

$$
S\left(t^{\prime}\right)=-i \frac{\hbar}{B} \frac{\partial}{\partial t^{\prime}}+H_{0}(\theta)+W\left(\theta, t^{\prime}\right),
$$

where the zeroth-order Hamiltonian is

$$
H_{0}(\theta)=\mathbf{J}^{2}-\omega_{S} \cos \theta-\frac{A}{T} \cos ^{2} \theta,
$$

and the perturbation operator is defined as

$$
W\left(\theta, t^{\prime}\right)=\frac{A}{T} \cos ^{2} \theta \sum_{n \neq 0} \exp \left[-(n \pi \sigma / T)^{2}+2 \pi i n t / T\right],
$$

where $A=\Delta \omega \int_{-T / 2}^{T / 2} \exp \left(-t^{\prime 2} / \sigma^{2}\right) d t^{\prime}$. Eigenstates of $H_{0}$ are the pendular states for a time-independent field given by the sum of the static field and the average value of the pulsed field over one period. On the other hand, the perturbation operator $W\left(\theta, t^{\prime}\right)$ has been obtained by Fourier expanding the pulse train. It represents a $t^{\prime}$-dependent interaction given by a sum of monochromatic components, which can mix significantly two eigenstates of $H_{0}\left(\psi_{i}^{0}\right.$ and $\left.\psi_{j}^{0}\right)$ only at accidental resonances, i.e., if $\Delta E_{i j} \cong 2 \pi n / B T$ and $\left\langle\psi_{j}^{0}\left|\cos ^{2} \theta\right| \psi_{i}^{0}\right\rangle \neq 0$. Thus, when the perturbation treatment is valid (for small $B T$ values) and in the absence of resonances, cyclic states of $S\left(t^{\prime}\right)$ will be similar to pendular states of $H_{0}$. Nonetheless, in general, a resonance will be always met, since $\Delta E_{i j}$ increases with $A / T$. If the resonance gives rise to strong mixture with higher states the orientation will not be recovered after the resonance is crossed.

Figure 1 illustrates the basic behavior of cyclic states as a function of the pulse area $A$. The behavior of eigenstates of $H_{0}$ is shown by continuous lines. In this limit the orientation increases with the ratio between the pulse area and the time delay between pulses, $A / T$. Thus, when $T$ increases the orientation is smaller for a given $A$. In general, a further increase of $A$ cannot compensate for the larger $T$ since reso- 


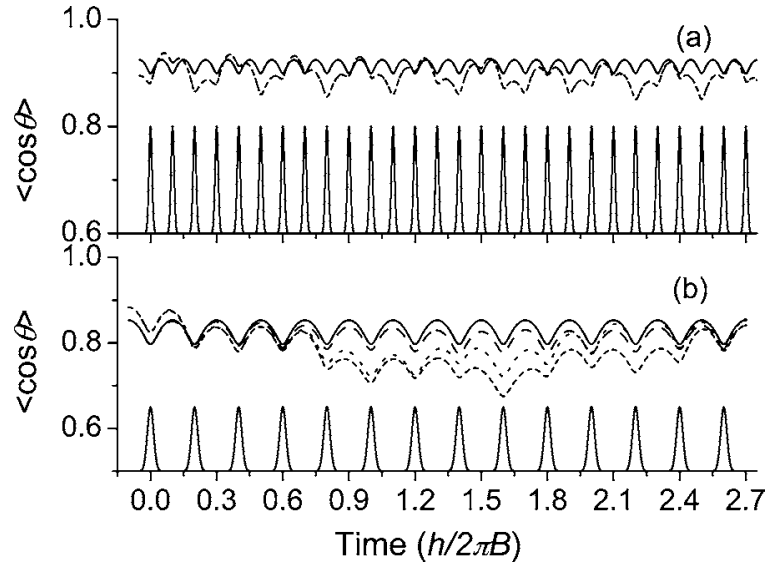

FIG. 2. The time evolution of the orientation for a molecule in a $\mathrm{dc}$ field combined with a periodic pulse train. (a) corresponds to 28 pulses with $\sigma=0.01, T=0.1, \Delta \omega=250$, and a static field interaction $\omega_{S}=0.1$. (b) corresponds to 14 pulses with $\sigma=0.02, T=0.2, \Delta \omega$ $=100$, and $\omega_{S}=0.5$. The orientation for exact cyclic states is given by solid lines and for switched wave packets by dotted lines. Orientation after removing the static field is given by dashed lines (exact cyclic states) and short dashed lines (switched wave packets). These curves are not visible in panel (a).

nances due to $W\left(\theta, t^{\prime}\right)$ arise, spoiling the orientation. For a fixed value of $A / T$, the perturbation $W\left(\theta, t^{\prime}\right)$ introduces another important point; a shortening of the pulse duration (smaller $\sigma$ ) requires a higher laser intensity to maintain orientation (in order to keep the same $A / T$ ). On the other hand, a smaller $\sigma / T$ ratio implies stronger resonances. Thus, for a given time delay $(T)$ between pulses a shorter pulse duration can deteriorate the maximum orientation achievable unless the resonances arise after the maximum is reached.

Figure 2 shows the time evolution of the orientation $(\langle\cos \theta\rangle)$ for cyclic states submitted to different pulse trains. Panel 2 (a) gives the orientation for a sequence of 28 pulses with $\sigma=0.01, T=0.1$, and $\Delta \omega=250$ in a static field with $\omega_{S}$ $=0.1$. For the molecule iodine monochloride, ICl $(\mu$ $=1.24 \mathrm{D}$, and $B=0.1142 \mathrm{~cm}^{-1}$ [4]) this corresponds to a static field of $548 \mathrm{~V} / \mathrm{cm}$, and a pulse duration of 0.8 ps. Orientation for longer pulses in shown in panel 2 (b) for $\omega_{S}$ $=0.5$.

\section{SWITCHED WAVE PACKETS}

The initial cyclic wave packet in Fig. 2(a) consists of a linear combination of seven $|J, M=0\rangle$ states with $J$ from 0 to 6 , and small contributions from higher excited states. In [16] it was argued that aligned cyclic states could be created by using tailored microwave pulses [23]. Here, we show that excellent approximations to oriented cyclic states can be created by a switched-wave-packet method $[18,19]$. The combination of an electrostatic field and a long laser pulse with a slow rising time guides adiabatically a molecule from a rotational eigenstate to an eigenstate of the instantaneous molecule-field Hamiltonian. Thus, at the field peak, for an initial eigenstate given by one of the lower rotational states, an oriented pendular state is created. If the laser is switched

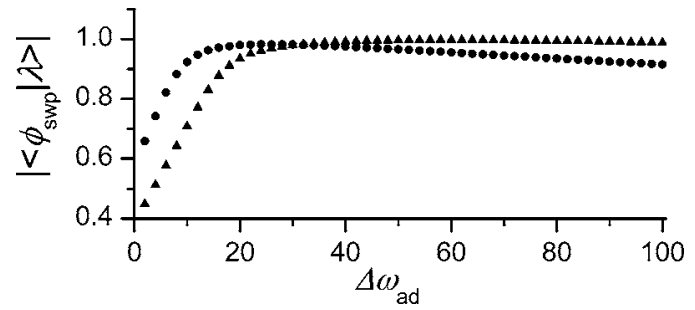

FIG. 3. The overlap between the initial cyclic states $(\lambda)$ of Fig. 2 and wave packets $\left(\phi_{\text {swp }}\right)$ obtained by suddenly turning off an adiabatic Gaussian pulse in a static field, as a function of the laser intensity $\left(\Delta \omega_{\mathrm{ad}}\right)$. Triangles correspond to the cyclic state of Fig. 2 (a), and circles to that in 2(b). Static fields are the same as in Fig. 2.

off suddenly (compared to the rotational period) the molecule will remain in the same rotational wave packet [18].

The connection between switched and cyclic wave packets can be understood by examining Eqs. (4) and (5). Eigenstates of $H_{0}$ correspond exactly to switched wave packets for a long pulse with a peak field corresponding to $\Delta \omega_{\mathrm{ad}}=A / T$. Thus, in the absence of resonances, cyclic states will be equivalent to switched wave packets. Once the resonance conditions are reached, cyclic states differ from the eigenstates of $H_{0}$ and consequently switched wave packets will give only approximations to them. The stronger the resonance the worse the approximation.

Figure 3 shows the overlap for the initial cyclic states of Fig. 2 and wave packets created by different switched pulses. There is a large overlap for a big range of laser intensities. Thus, for the cyclic state corresponding to the pulse with $T$ $=0.1$ the overlap is greater than 0.95 for $20 \leqslant \Delta \omega_{\mathrm{ad}} \leqslant 100$. This saturation in the overlap implies that pendular states change very little once they are strongly localized. Further optimization of the switched pulse would improve the maximum overlap with the exact cyclic state but our interest is to show the robustness of the method by using states that can be easily created and resemble the exact cyclic wave packets.

Figure 2 also shows the time evolution of the orientation for switched wave packets. The case shown in 2(a) illustrates a practically perfect case. The dotted curve pertains to a switched wave packet for $\Delta \omega_{\mathrm{ad}}=30\left[\left|\left\langle\phi_{\mathrm{swp}} \mid \lambda\left(t_{0}\right)\right\rangle\right|=0.97\right]$ and it practically coincides with the curve corresponding to the exact cyclic state. Maximum overlap occurs for $\Delta \omega_{\mathrm{ad}}$ $=60$ (if $W=0$ maximum overlap should occur for $\Delta \omega_{\text {ad }}$ $=44.3$ ).

\section{FIELD-FREE ORIENTATION}

Our choice of cyclic wave packet as initial states offers a crucial advantage over previous schemes based on submitting a rotational eigenstate to an adiabatic pulse. A long pulse only can lead to orientation inside the dc field. When the pulse ends molecules return adiabatically to their initial states, i.e., the Stark states due to the dc field, which, at the best, are only moderately orientated [for the case shown in Fig. 2(a), $\left.\omega_{S}=0.1,\langle\cos \theta\rangle \approx 0\right]$. On the other hand, with our approach it is possible to conserve high molecular orientation after extraction of molecules from the dc field. Figure 4 


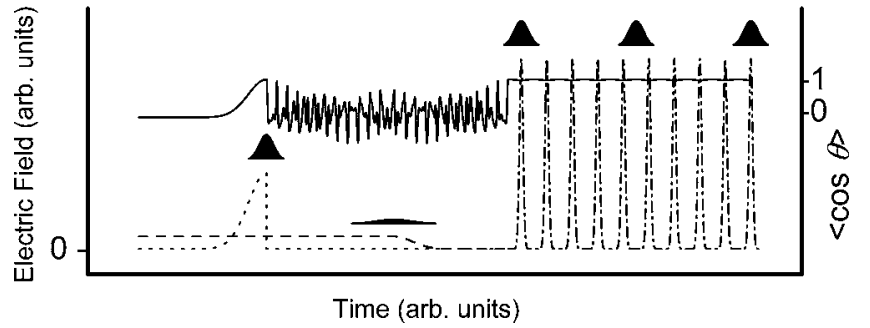

FIG. 4. A sketch of the fields (not scaled) employed in the orientation scheme. The dashed line is the static field. The dotted line is the switched adiabatic laser pulse. The dash-dotted line is the pulse train. The wave packets become strongly localized in the polar angle $\theta$ at the end of the switched pulse, and remain localized during the pulse train. During the time interval needed to switch off the static field the wave packet spreads due to dephasing, but for small $\omega_{S}$ the wave packet can be reconstructed before the pulse trains starts. A pictorial representation (solid line) of the evolution of orientation during the procedure is also given.

is a sketch of the different processes involved in our scheme. A molecule initially placed in a rotational eigenstate is guided by a switched pulse to a cyclic wave packet. This oriented state can be written as a linear superposition of field-free rotor eigenstates $\psi\left(t_{i}\right)=\Sigma_{n} c_{n}|J\rangle_{n}$ or alternatively as a superposition of Stark states of the molecule in the dc field, $\psi\left(t_{i}\right)=\Sigma_{n} d_{n}|\widetilde{J}\rangle_{n}$. If $\omega_{S}$ is small each Stark state consists of one rotational eigenstate and very small contributions of other eigenstates, i.e., $|\widetilde{J}\rangle_{n} \cong|J\rangle_{n}$ holds, and consequently $d_{n} \cong c_{n}$ for all $n$.

Additionally, the dipole interaction, for diatomic or linear molecules, can be adiabatically switched off by turning off the electrostatic field with a fast switch [24], in a process that may take a few nanoseconds. During the time interval needed to zero the static field, each Stark state contributing to $\psi\left(t_{i}\right)$ evolves adiabatically and accumulates a phase factor, $\left|\widetilde{J}_{n}\right\rangle \rightarrow \exp \left(-i \alpha_{n}\right)|J\rangle_{n}$. Thus, remembering that $d_{n} \cong c_{n}$ for small $\omega_{S}$, the wave function after zeroing the static field is $\psi(t) \cong \Sigma_{n} c_{n} \exp \left(-i \alpha_{n}\right)|J\rangle$. In other words, the modulus of the contribution of the different rotational eigenstates to the final wave function is approximately the same as in the oriented cyclic state. Therefore, the oriented cyclic state could be approximately rebuilt outside the static field if the phase factors of the different components of the wave packet can be controlled. Plausibly, an electric-field path can be designed for which $\alpha_{n}$ is nearly the same for all $n$. This requires manipulating the shape of the electrostatic field and the time interval needed to switching it off (see the deep analysis, by Friedrich [24], of the control that can be exerted over the fields arising in a Stark decelerator).

Once the oriented cyclic state is rebuilt, the same periodic pulse train that kept molecules oriented in the dc field can be switched on (now without the influence of the static field) to maintain orientation. This procedure requires that the oriented cyclic state corresponds to a small value of $\omega_{S}$. Under this condition, a cyclic state, $\lambda$, of $S(t)[\mathrm{Eq} .(2)]$ is well approximated by a linear combination of two quasidegener- ate aligned cyclic states; $\lambda_{a}^{0}$ for even $J$ 's, and $\lambda_{b}^{0}$ for odd $J$ 's, of the same $\left(t, t^{\prime}\right)$ Hamiltonian with $\omega_{S}=0$. The wave function, at the end of a period, is therefore given by

$$
U(T / 2,-T / 2)|\lambda\rangle \cong \exp \left(-i \bar{\epsilon}_{\lambda} T\right) \frac{\left|\lambda_{a}^{0}\right\rangle+\left|\lambda_{b}^{0}\right\rangle}{\sqrt{2}},
$$

with $\bar{\epsilon}_{\lambda}=\left(\epsilon_{\lambda_{a}^{\prime}}+\epsilon_{\lambda_{b}^{\prime}}\right) / 2$. In other words, the cyclic state for the $\left(t, t^{\prime}\right)$ operator that includes a weak dipole interaction is an approximate cyclic state for the operator with no static field. Figure 2 shows the time evolution of the orientation for two cases. For 2(a) the orientation is indistinguishable from that obtained with the static field on. For 2(b) Eq. (6) does not hold, and the orientation is slightly lost for the central pulses.

\section{CONCLUSIONS}

We have devised a scheme that makes it possible to maintain a large molecular orientation during an arbitrary time. The method is based on submitting rotational wave packets in an electrostatic field to the periodic sequence of nonresonant laser pulses for which they are cyclic states. Also, we have discussed some speculative ideas that would permit to remove the static field and conserve large orientation. Thus, this control scheme would allow us to obtain periodically (between pulses) large and nearly constant field-free orientation.

The previous results hold for molecules with a minute dipole moment. Thus, the use of trains of nonresonant pulses offers an advantageous alternative to methods based on the use of trains of half-cycle pulses, that require a large dipole moment. The orientation shown in Fig. 2(a) $(\langle\cos \theta\rangle \approx 0.92)$ could be obtained for a typical molecule $\left(B=0.1 \mathrm{~cm}^{-1}\right)$, using electrostatic fields attainable in the laboratory $(120 \mathrm{kV} / \mathrm{cm})$, for a dipole moment as little as $0.005 \mathrm{D}$.

On the other hand, a connection between cyclic wave packets and switched wave packets has been found. This gives an easy way to create appropriate initial states to implement our strategy. A long nonresonant pulse suddenly switched off is able to create excellent approximations to the exact cyclic states.

This strategy can be readily implemented for polyatomic molecules. However, orientation with no dc field, could be impossible for asymmetric tops. Contrary to linear molecules, asymmetric-top Stark energy levels can undergo sharp avoided crossing as a function of electric field strength [3]. Thus, molecules can experience, even for a slowly varying time-dependent field, nonadiabatic evolution, and therefore oriented states could not be reconstructed outside the electrostatic field.

\section{ACKNOWLEDGMENTS}

We are grateful to J. M. Fernández and J. L. Doménech for helpful comments on the manuscript. Financial support from the Ministerio de Educación y Ciencia of Spain, under Project No. FIS2004-02558 is acknowledged. 
[1] H. Stapelfeldt and T. Seideman, Rev. Mod. Phys. 75, 543 (2003).

[2] H. J. Loesch and J. Remscheid, J. Chem. Phys. 93, 4779 (1990); B. Friedrich and D. R. Herschbach, Z. Phys. D: At., Mol. Clusters 18, 153 (1991).

[3] R. Escribano, B. Maté, F. Ortigoso, and J. Ortigoso, Phys. Rev. A 62, 023407 (2000).

[4] B. Friedrich and D. Herschbach, J. Chem. Phys. 111, 6157 (1999); J. Phys. Chem. A 103, 10280 (1999).

[5] R. Baumfalk, N. H. Nahler, and U. Buck, J. Chem. Phys. 114, 4755 (2001); S. Minemoto et al., ibid. 118, 4052 (2003); H. Sakai, S. Minemoto, H. Nanjo, H. Tanji, and T. Suzuki, Phys. Rev. Lett. 90, 083001 (2003).

[6] S. Guérin, L. P. Yatsenko, H. R. Jauslin, O. Faucher, and B. Lavorel, Phys. Rev. Lett. 88, 233601 (2002).

[7] J. Ortigoso, M. Rodríguez, M. Gupta, and B. Friedrich, J. Chem. Phys. 110, 3870 (1999).

[8] L. Cai, J. Marango, and B. Friedrich, Phys. Rev. Lett. 86, 775 (2001).

[9] M. J. J. Vrakking and S. Stolte, Chem. Phys. Lett. 271, 209 (1997).

[10] M. Machholm and N. E. Henriksen, Phys. Rev. Lett. 87, 193001 (2001); C. M. Dion, A. Keller, and O. Atabek, Eur. Phys. J. D 14, 249 (2001).

[11] D. Sugny, A. Keller, O. Atabek, D. Daems, C. M. Dion, S.
Guérin, and H. R. Jauslin, Phys. Rev. A 69, 033402 (2004).

[12] C. M. Dion, A. D. Bandrauk, O. Atabek, A. Keller, H. Umeda, and Y. Fujimura, Chem. Phys. Lett. 302, 215 (1999).

[13] D. Daems, S. Guérin, D. Sugny, and H. R. Jauslin, Phys. Rev. Lett. 94, 153003 (2005).

[14] P. Marquetand, A. Materny, N. E. Henriksen, and V. Engel, J. Chem. Phys. 120, 5871 (2004).

[15] M. Leibscher, I. Sh. Averbukh, and H. Rabitz, Phys. Rev. Lett. 90, 213001 (2003); Phys. Rev. A 69, 013402 (2004).

[16] J. Ortigoso, Phys. Rev. Lett. 93, 073001 (2004).

[17] U. Peskin and N. Moiseyev, J. Chem. Phys. 99, 4590 (1993).

[18] T. Seideman, J. Chem. Phys. 115, 5965 (2001).

[19] J. G. Underwood, M. Spanner, M. Yu. Ivanov, J. Mottershead, B. J. Sussman, and A. Stolow, Phys. Rev. Lett. 90, 223001 (2003).

[20] A. N. Seleznyova, J. Phys. A 26, 981 (1993); G. Jolicard and J. P. Killingbeck, J. Phys. A 36, R411 (2003).

[21] T. Seideman, Phys. Rev. Lett. 83, 4971 (1999).

[22] I. Vorobeichik and N. Moiseyev, Phys. Rev. A 59, 2511 (1999); J. Ortigoso, ibid. 70, 055401 (2004).

[23] R. S. Judson, K. K. Lehmann, H. Rabitz, and W. S. Warren, J. Mol. Struct. 223, 425 (1990); J. Ortigoso, Phys. Rev. A 57, 4592 (1998).

[24] B. Friedrich, Eur. Phys. J. D 31, 313 (2004). 